\title{
EL CARÁCTER ARGUMENTATIVO DEL DERECHO: UNA DEFENSA DEL POST-POSITIVISMO DE MACCORMICK *
}

\author{
Thomas da Rosa de Bustamante ** \\ Universidad Federal de Minas Gerais (Brasil)
}

RESUMEN. En los escritos de N. MACCormick, existe tanto una teoría del derecho -la denominada teoría institucional — como una teoría de la argumentación jurídica. La atención que MACCORMICK presta a la justificación de las decisiones jurídicas le hace plantear asuntos que no pueden ser contestados por una teoría positivista del derecho. Esta es, claramente, una de las razones por las cuales este autor defendió abiertamente, en sus últimos trabajos, una teoría post-positivista del derecho. En este ensayo, dicha teoría es comparada con el positivismo contemporáneo, que puede ser ejemplificado por medio de la teoría jurídica de J. RAZ. Se concluye, después de un breve análisis de las tesis centrales del positivismo y de algunos argumentos de MACCORMICK que lo apartan de estas tesis, que el post-positivismo es superior a todas las versiones del positivismo jurídico. En el caso específico de MACCORMICK, los siguientes argumentos son contribuciones relevantes para el desarrollo de la ciencia del derecho más allá de los límites del positivismo jurídico: 1) la caracterización del derecho como un concepto "argumentativo»; 2) la teoría reflexiva de las fuentes del derecho, adoptada por MACCORMICK; 3) la concepción kantiana de la justificación jurídica que da fundamento a su teoría de la argumentación jurídica; 4) la tesis del caso especial; 5) la tesis de la pretensión de corrección del derecho y la obligación, que se deriva de ella, de hacer justicia conforme el derecho, y 6) la tarea desempeñada por la eticidad y por la moralidad política en la creación y en la aplicación del derecho.

Palabras clave: MACCORMICK, post-positivismo, tesis de las fuentes sociales, teoría de la argumentación jurídica.

ABSTRACT. In N. MACCORMICK's legal writings there is both a legal theory -the so-called institutional theory - and a theory of legal argumentation. His concern with the justification of legal decisions has made him ask questions which cannot be answered by a positivistic theory of law. This is, beyond any doubt, one of the reasons why this author has openly advocated a post-positivistic theory of law. In this paper, this theory is contrasted with contemporary positivism, which can be exemplified by J. RAz's juridical writings. I conclude, after a brief analysis of the central theses of J. RAZ and of some of the theses advanced by MACCORMICK against positivism, that his postpositivism is a superior form of legal theory. In the case of MACCORMICK, the following points are both relevant for the development of legal science and incompatible with positivism: 1) his characterization of law as an argumentative concept; 2) his critical theory of the sources of law; 3 ) his Kantian conception of legal justification and his theory of legal argumentation; 4) the special case thesis; 5) the thesis of the claim to correctness and the obligation to do justice in accordance with the law; 6 ) and the role played by the ethical life of the community and by the political morality in the development and application of law.

Keywords: MACCORMICK, post-positivism, social sources thesis, theory of legal argumentation.

* Fecha de recepción: 12 de septiembre de 2012. Fecha de aceptación: 15 de octubre de 2012.

** Profesor y miembro del cuerpo docente permanente del Programa de Postgrado en Derecho de la Universidad Federal de Minas Gerais. Doctor en derecho de la Pontificia Universidad Católica de Rio de Janeiro. Contacto: tbustamante@ufmg.br. 


\section{INTRODUCCIÓN}

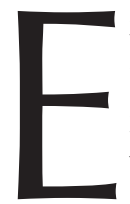

n este ensayo se investiga la plausibilidad de la aserción de que el derecho es argumentativo, la cual está situada en el núcleo del post-positivismo de N. MacCormick. En nuestra interpretación de los trabajos de este autor, la insistencia del positivismo en la pretensión de que la validez de una ley puede ser identificada sólo por sus características formales — de suerte que el teórico del derecho debe limitarse a identificar la «fuente» de una norma jurídica para afirmar su validez - siempre fue incoherente con la teoría de la argumentación jurídica de MACCORMicK, incluso en su versión inicial.

Quizás la forma más fácil de entender eso sea comparar el post-positivismo de MACCORMICK con el positivismo excluyente de J. RAZ, que es la concepción positivista más radical sobre el carácter del derecho como hecho social y el método para atribuirle a una norma particular el status de una regla jurídica válida. El hecho de que la mayor parte de los positivistas incluyentes han desistido de su tesis original de que es posible una conexión contingente entre el derecho y la moral, es una buena razón para empezar nuestra argumentación con los trabajos de RAZ, que pueden ser descritos como la versión más robusta del positivismo jurídico contemporáneo.

Mi camino será el siguiente. En la primera sección, que es substancialmente menos extensa que la segunda, analizo algunas de las tesis de RAZ sobre la naturaleza del derecho. Estas tesis, según pienso, son aceptadas por las vertientes más importantes del positivismo contemporáneo. Ellas pueden suponer, por tanto, un punto de partida para cualquier investigación post-positivista sobre la naturaleza del derecho. En la segunda sección, por su parte, intento subrayar las afirmaciones centrales del post-positivismo de MACCORMICK, que parecen ser suficientes para que podamos abandonar el positivismo como proyecto teórico.

\section{CONSIDERACIONES SOBRE LOS ASPECTOS CENTRALES DEL POSITIVISMO CONTEMPORÁNEO}

Pese al debate entre los positivistas incluyentes y los positivistas excluyentes, referido a las diferentes interpretaciones de las «Tesis de la Separabilidad» entre el derecho y la moral, me parece que los positivistas contemporáneos hoy día tienen una nueva agenda para la teoría jurídica, que se refiere a la denominada «Tesis de las Fuentes Sociales». Quizás la formulación más exacta de esta tesis sea la elaborada por J. RAZ, que pone particular énfasis en la idea de que «todo el derecho está basado en alguna fuente», ya que «su existencia y contenido pueden ser identificados tomándose como referencia hechos sociales, sin recurrir a cualquier enunciado evaluativo» (RAZ, 1994: 195).

Es probable que la forma más sencilla de comprender la concepción de RAZ sobre el derecho sea retomar su distinción entre las dos fases de la argumentación práctica. Cuando consideramos la actitud de una persona respecto de una cierta acción propuesta, podemos diferenciar entre la etapa deliberativa y la etapa ejecutiva. En instituciones como el Estado, por ejemplo, frecuentemente es el caso de que algunos actores 
son competentes para tomar decisiones, mientras que otros deben meramente ejecutar las decisiones tomadas por terceros. En la etapa deliberativa, según RAZ, «la cuestión sobre lo que debe hacerse está abierta a argumentos basados en todos los tipos de consideraciones», incluso razones morales, mientras que en la etapa ejecutiva la cuestión sobre lo que debe hacerse «será identificada sin recurrir a ulteriores argumentaciones morales», pues sólo consideraciones «positivas» pueden pertenecer a este sector de la racionalidad práctica (RAZ, 1994: 191).

Cuando pensamos en las instituciones jurídicas, la principal premisa que caracteriza la teoría de RAZ como positivista es la tesis de que el derecho «consiste únicamente en consideraciones positivas dotadas de autoridad». Esta presuposición desplaza todas las consideraciones morales, éticas, políticas y también pragmáticas hacia fuera de las fronteras del derecho y de la argumentación jurídica. Según RAZ, «consideraciones positivas son aquellas cuya existencia y contenido pueden ser aseveradas sin recurrir a valoraciones morales» (RAZ, 1994: 189). Puede decirse, por tanto, que para RAZ el derecho no es más que una autoridad institucionalizada.

Esta concepción estricta del derecho supone un ámbito restringido para el razonamiento jurídico y para la teoría del derecho. Un juez puede muy bien ser capaz de dejarse influenciar por consideraciones morales o cualquier otro tipo de consideraciones no-positivistas, pero cuando lo hace ya no está aplicando el derecho. Por el contrario, estará ejerciendo su competencia para crear una nueva regla jurídica, aunque dicha regla sea contraria al sentido literal de un enunciado normativo preexistente. Esta demarcación de los límites entre la aplicación y la creación del derecho es muy clara en la teoría jurídica de RAz.

La esencia del positivismo jurídico contemporáneo, por tanto, está plasmada en la pretensión de que los criterios de legalidad son «independientes de su contenido» (content-independent), pues las denominadas «consideraciones positivas» serían suficientes para establecer la validez de cualquier regla jurídica particular. En efecto, incluso las figuras más prominentes del positivismo incluyente parecen haber abandonado ya su tesis original de que son posibles conexiones contingentes entre el derecho y la moral y pasan a reconocer que la versión de positivismo jurídico desarrollada por RAZ es más interesante que aquella anteriormente sostenida por esos autores. Para expresar esa idea en términos literales, cito el siguiente extracto de un artículo reciente de J. COLEMAN:

Aunque la visión convencional sea que el positivismo es definido por medio de la tesis de la separabilidad, la concepción más precisa es la de que el positivismo se define por su comprometimiento con la tesis de los hechos sociales. Una afirmación familiar de la tesis de los hechos sociales es la que Raz denomina de tese de las «Fuentes Sociales». De acuerdo con esta tesis, la identidad y el contenido del derecho puede ser determinado únicamente por hechos sociales (COLEMAN, 2007: 586).

Podemos percibir, claramente, por tanto, que esta visión constituye un cambio significativo en la teoría jurídica de COLEMAN, pues su positivismo incluyente no puede ser establecido sin la Tesis de la Separabilidad. El autor parece claramente abandonar, por tanto, el positivismo incluyente que él mismo creó.

Sin el positivismo incluyente, las teorías jurídicas positivistas parecen retroceder a lo que BOBBIO entendía que era la esencia del positivismo metodológico, es decir, a la 
tesis de que la validez del derecho no depende de su contenido, y de que el derecho debe ser estudiado como es, y no como sus estudiosos piensen que deba ser (BOBBIO, 1998: 23).

Uno de los modos de definir el post-positivismo, por tanto, es analizar las tesis que sus proponentes sostienen contra la Tesis de las Fuentes Sociales, pues basta que se descarte esta tesis para que se pueda definir a alguien como no-positivista o, si la persona en cuestión pretende apartarse también del iusnaturalismo, como post-positivista.

En las secciones siguientes, se hará un análisis del post-positivismo de N. MACCormicK a fin de demostrar que su teoría transciende los límites del positivismo. Se sostendrá, en lo siguiente, que el carácter argumentativo del derecho y las conexiones entre la argumentación jurídica y el discurso práctico no pueden ser reconciliados con el positivismo, y por tanto implican la necesidad de buscar un nuevo tipo de teoría del derecho.

\section{EL DERECHO, LA MORAL Y LA ARGUMENTACIÓN: LA NATURALEZA DEL POST-POSITIVISMO DE MACCORMICK}

Aunque MACCORMiCK sólo adopte el rótulo de post-positivista en los últimos años de su carrera, su relación con el positivismo nunca ha estado libre de problemas. Incluso en sus escritos iniciales el autor nunca estuvo satisfecho con la posición positivista sobre la naturaleza del derecho. Pese a la influencia de HART sobre sus trabajos, siempre hubo críticas por parte del autor a la neutralidad científica absoluta de los proyectos teóricos de HART y KELSEN. Quizás el más expresivo de estos desacuerdos se refiera a la cuestión de la justificación de las decisiones sobre la validez de las normas jurídicas, consideradas desde la perspectiva interna. Como MACCORMICK enfatizó repetidamente, los teóricos del derecho deben, según su pensamiento, llevar el insight de HART sobre el punto de vista interno más allá de donde HART lo dejó en sus escritos teóricos ${ }^{1}$. Cuando los teóricos del derecho tienen en consideración todas las implicaciones del punto de vista interno, el problema de la justificación de las decisiones jurídicas se torna central, como se puede leer en el siguiente pasaje de los escritos iniciales de MACCORMICK:

Una descripción positivista del sistema tal como opera no puede contestar a un tipo particular de interrogación que puede ser formulada internamente en un sistema jurídico: la cuestión puede ser formulada ante un juez, en un caso difícil, de la siguiente manera: «¿Por qué nosotros debemos tratar todas las decisiones alcanzadas en conformidad con una regla válida según nuestro criterio de validez como suficientemente justificadas?», y este es un interrogante que ocasionalmente puede ser planteado, y de tiempos en tiempos lo es. Además, el positivismo tampoco logra contestar a la cuestión planteada por los jueces con aún

1 Para ilustrar este punto, puede mencionarse el siguiente fragmento de MACCORMICK en una entrevista concedida a M. ATIENZA en la ocasión de publicación de su libro Institutions of Law: «El aspecto en el que la obra de HART resulta más iluminadora y perdurable se refiere a la necesidad de entender la conducta gobernada por reglas desde el "punto de vista interno". Esto es esencial para desarrollar una teoría clara y convincente de las normas; pero las reglas son sólo un tipo de normas. El análisis del derecho como la unión de reglas primarias y secundarias, aunque lleno de intuiciones valiosas, es, al final, incompleto e insatisfactorio. Se necesita un nuevo comienzo» (ATIENZA, 2006: 482). 
más frecuencia: ¿Cómo debemos justificar las decisiones concernientes a la interpretación y a la aplicación de nuestros criterios de validez? (MACCORMicK, 1978: 63).

Esta cita demuestra que MACCORMICK está preocupado con algo que es normalmente olvidado por el positivismo contemporáneo: el problema de la justificación de las decisiones jurídicas. Está especialmente preocupado con la justificación de las decisiones jurídicas porque es consciente del elemento subjetivo que está siempre presente, en mayor o menor medida, en las actividades de interpretación y de aplicación del derecho. Además, asume el punto de vista de un «usuario» de la norma cuando reivindica para sí la tarea de construir una explicación teórica de la naturaleza del derecho.

Aunque su teoría institucional del derecho, la cual es una teoría jurídica en sentido estricto, establezca una diferencia entre el punto de vista del teórico del derecho y el de los juristas prácticos, la investigación del teórico del derecho queda «orientada por valores» cuando hace descripciones de las instituciones jurídicas (MACCORMICK, 2007: 301). Según MACCORMICK, «una explicación coherente de la naturaleza del derecho, y una explicación coherente del carácter de cualquier sistema jurídico moderno, debe tomar en serio los valores muy generales que son inherentes a la naturaleza del fenómeno jurídico». Los conceptos que un teórico del derecho emplea para explicar la naturaleza del derecho son, por tanto, «conceptos interpretativos» en el sentido de R. DwORKIN, pues el teórico debe adoptar un punto de vista hermenéutico que pretende «comprender las prácticas e instituciones de los seres humanos según lo que las hace inteligibles y válidas [...] para sus participantes» (MACCORMICK, 2007: 295).

Si, por una parte, el teórico tiene un cierto grado de distanciamiento respecto de los «actores centrales» de la práctica jurídica, debe tener, como un actor de segundo nivel, «un grado relativamente alto de compromiso, debidamente contrastado con los observadores externos» (MACCORMICK, 2007: 7). Aunque MACCORMICK sostenga que su teoría del derecho (jurisprudence) no tiene por objetivo solucionar problemas prácticos particulares (MACCORMICK, 2007: 302), la comprensión que esta ofrece para la categoría práctica del «derecho» presupone una reconstrucción racional de las instituciones jurídicas que «busca una consideración crítica de las reglas centrales a la luz de los principios y valores que subyacen a ellas». Dicha consideración crítica, según MACCORMICK, «puede indicar la dirección de futuras interpretaciones del derecho que rectificarán anomalías en el entendimiento corriente, incluso en la práctica judicial» (MACCORMICK, 2007: 292) ${ }^{2}$.

A esta comprensión de la investigación jurídico-teórica como predominantemente crítica y orientada por valores, adoptada por MACCORMICK, se adiciona la concepción de la práctica de la argumentación jurídica como un proceso también necesariamente constructivo y hermenéutico, que parece no ser compatible con el esencialismo empirista que caracteriza la mayoría de las concepciones positivistas.

Para muchos positivistas el derecho es una especie de entidad real que está ahí para ser «conocido» o «descubierto» por el teórico del derecho. Los teóricos positivistas del derecho raramente participan de una actividad genuinamente interpretativa o hermenéutica cuando intentan «identificar» el derecho. El derecho posee, para ellos,

2 Así como AleXy, MACCORMiCK sostiene que la ciencia del derecho no tiene sólo una dimensión empírica y una dimensión analítica, sino también una dimensión normativa (MACCORMICK, 2007: 292). 
una esencia fáctica que puede ser observada y descrita desde el exterior. El teórico mira al derecho desde el punto de vista externo, pues su contenido ya está fijado y su significado no depende de las subjetividades del intérprete. Este tipo de positivismo, que es largamente dominante en la teoría jurídica anglo-americana, sostiene que hay una separación entre la «creación» y la «aplicación» del derecho. Se puede identificar empíricamente el «derecho válido» en una sociedad dada, pese el hecho de que el juez eventualmente puede «crear» un nuevo derecho cuando no estuviera satisfecho con la solución ofrecida por el sistema jurídico o, más frecuentemente, cuando estuviera enfrentando un caso todavía no solucionado por el conjunto de las reglas jurídicamente válidas. Es exactamente de este tipo de positivismo del que hablamos cuando pensamos en J. RAZ, para quien es posible «aplicar» el derecho sin ninguna ponderación de razones. La denominada «aplicación del derecho» es únicamente la etapa ejecutiva de la argumentación práctica. Todo el derecho puede ser encontrado en sus fuentes sociales, y la principal tarea del jurista es identificar esas fuentes, pues éstas le dan una orientación acerca de lo que se está obligado a hacer ante un caso particular.

Dicha diferenciación clara entre la creación y la aplicación del derecho lleva a otra distinción que es también típica del positivismo anglo-americano contemporáneo: la distinción entre «teorías del derecho» y «teorías de la decisión jurídica» (theories of adjudication). Las teorías del derecho conciernen en general a la identificación del derecho. Contestan a la cuestión acerca de lo que el derecho es, y no de lo que el derecho debe ser. Debe haber un único test para diferenciar las reglas jurídicas de los otros tipos de normas sociales, y este test normalmente tiene que ver con su pedigrí o con el proceso por medio del cual esas reglas son creadas (en otros términos, con sus fuentes). Desde esta perspectiva, el derecho es auto-referencial puesto que regula el proceso por medio del cual las normas jurídicas son creadas. Esta distinción entre «teorías del derecho»y «teorías de la decisión» supone un elemento positivista. En la realidad, difícilmente es posible imaginar un no-positivista que estuviera dispuesto a aceptar esta separación.

La diferencia entre una teoría del derecho (theory of law) y una teoría de la decisión (theory of adjudication) es crucial para el debate sobre el positivismo contemporáneo, ya que ilustra cómo este tipo de teoría caracteriza la función de la ciencia del derecho como disciplina teórica. Si la ciencia del derecho es clasificada como una teoría del derecho, por oposición a una teoría de la decisión, puede notarse un creciente abismo entre la teoría y la práctica, que son concebidas como contextos discursivos autónomos que raramente se comunican uno con otro. No es tarea de un teórico justificar cualquier práctica o decisión particular, e incluso proporcionar cualquier criterio para la interpretación adecuada y la aplicación del derecho. La tarea propia de la teoría jurídica es meramente explicar el derecho, en lugar de desarrollarlo o revisarlo. Un buen teórico del derecho debe, por tanto, dejar su objeto de investigación intacto. La teoría jurídica y la práctica jurídica son terrenos diferentes, que deben permanecer separados en la medida en que estén destinados a cumplir con sus papeles sociales.

Como se explicará a continuación, la versión particular del post-positivismo defendida por MACCORMICK tiene como punto de partida dichas distinciones y, por tanto, conduce a una concepción del derecho más rica, pues no acepta la estrecha separación positivista entre el derecho tal como es y el derecho tal como debe ser, y tampoco la 
distinción entre las teorías del derecho y las teorías de la decisión. Además, también rechaza la tesis según la cual las fuentes del derecho son exhaustivas y suficientes para determinar el contenido de las reglas jurídicas.

El derecho es comprendido como una práctica interpretativa en la cual el sentido de sus normas es gradualmente construido, y no descubierto por la mera observación de fenómenos empíricos. El derecho como práctica social es inherentemente argumentativo, y una de las características más importantes de la argumentación jurídica es que permanece abierta a argumentos morales, éticos y pragmáticos que están involucrados en el discurso jurídico. En efecto, el discurso jurídico es entendido por MACCORMiCK como un caso especial de discurso práctico, y eso es suficiente para que podamos decir que la propia naturaleza de la argumentación jurídica crea una obligación de decidir de modo moralmente correcto, o, en las palabras de MACCORMICK, una obligación de «hacerse justicia conforme al derecho».

\subsection{El carácter argumentativo del derecho}

El primer aspecto digno de mención en la teoría institucional de MACCORMICK es que el derecho comprende más que un conjunto de hechos institucionales que no necesitan de interpretación ulterior. Como orden normativo, y «como un orden práctico», el derecho está «en constante necesidad de adaptación a nuevos problemas prácticos» (MACCORMICK, 2005: 6). En este particular, MACCORMICK acepta la idea kelseniana de que el derecho presenta una estructura jerárquica en la cual las normas de rango más alto son individualizadas y concretadas en los procesos de legislación y de aplicación del derecho. En el derecho comunitario europeo, por ejemplo, el proceso de transposición del nivel del derecho supranacional para el nivel de los ordenes jurídicos domésticos «es parte del Stufenbau, es decir, del proceso que camina paso a paso de la norma general para las decisiones particulares de casos concretos» (MACCORMICK, 2005: 10). Como MACCORMICK escribe con claridad, él ve al derecho como «una disciplina argumentativa», y no como una «ciencia exacta». Una de las características más esenciales del derecho es su naturaleza cuestionable (MACCORMICK, 2005: 14-15). A diferencia de los positivistas, que erigen la certeza del derecho a la condición de único valor asegurado por el principio del Estado de derecho, MACCORMICK acredita que el «carácter argumentativo del derecho» es algo para ser celebrado en sociedades democráticas, pues él está profundamente convencido de la idea de Estado de derecho. El reconocimiento del Estado de derecho como idea política implica el reconocimiento del derecho como el locus de la argumentación (MACCORMicK, 2005: 13). Aunque el principio del Estado de derecho esté orientado hacia el valor de la certeza del derecho, este valor no es el único. Racionalidad y justicia también figuran entre los valores básicos que constituyen el ideal fundamental del Estado de derecho.

En esta interpretación, la indeterminación del derecho no es algo de lo que lamentarse. Tiene relación con la idea de Estado de derecho y con las reglas procesales de argumentación que se encuentran implicadas en la estructura institucional que establece. Las ideas de imparcialidad y de equidad entre las partes en una disputa jurídica, bien como el principio fundamental consubstanciado en el adagio audiatur et altera pars, están necesariamente conectados con la idea fundamental del Estado de derecho 
y con el carácter argumentativo del sistema jurídico. En este sentido, merece la pena mencionar las siguientes palabras de MACCORMICK en el primer capítulo de la última versión de su teoría de la argumentación jurídica:

Creo en el carácter argumentativo del derecho, y considero eso admirable en una sociedad abierta. Debemos tomar en cuenta todos los aspectos de una cuestión importante, y no simplemente tomar parte por nuestros preconceptos o la certeza aparente. Debemos escuchar todos los argumentos, y celebrar, no lamentar, la naturaleza discutible que parece estar incorporada en el derecho (MACCORMICK, 2005: 16).

El propio principio del Estado de derecho, según MACCORMICK, implica un cierto grado de indeterminación en el sistema jurídico. Esta indeterminación, como lo explica el autor, no es sólo «el resultado del hecho de que los Estados comunican sus materiales jurídicos en el leguaje (oficial) natural, y esta padece de ambigüedad, vaguedad o textura abierta», como sostenía HART (MACCORMICK, 2005: 26). Esta también resulta del «debido reconocimiento de los "derechos de defensa" en cualquier sistema de persecución penal y contencioso civil» (MACCORMICK, 2007: 26). Dicho con brevedad, el Estado de derecho implica y, en cierto sentido, amplifica el carácter discutible del derecho.

Si esta interpretación del ideal político del Estado de derecho es correcta, entonces los teóricos del derecho tradicionales están equivocados cuando presentan el valor de la certeza jurídica como el único contenido del Estado de derecho. Además, los teóricos están también equivocados cuando sostienen, como lo hace RAZ, que el derecho debe ser «encontrado» en un conjunto previamente determinado de «fuentes sociales», por medio de una argumentación puramente empírica. La validez y el sentido de una ley no puede ser meramente una cuestión de hecho, sino que, al contrario, precisa ser al menos en cierta medida una cuestión de argumentación. En la perspectiva de MACCORMICK, el derecho muy raramente esta «fijado» (settled) y sus reglas son necesariamente derrotables, pues están inevitablemente sujetas a interpretaciones constructivas que pueden llevar a revisiones, reinterpretaciones e incluso a excepciones en sus supuestos de hecho. Las reglas jurídicas son vislumbradas como enunciando únicamente condiciones «ordinariamente necesarias y presuntamente suficientes» para las situaciones que regulan, ya que sus principios subyacentes, que proveen la justificación general del sistema jurídico, interaccionan con las provisiones más específicas que se encuentran en la legislación, en los precedentes y en la legislación secundaria (MACCORMICK, 2005: 241). Esta interacción puede, eventualmente, activar algún «antecedente» (background factor) cubierto por el principio justificador de la regla jurídica, que puede llevar al reconocimiento de excepciones no escritas a la regla inicialmente considerada por el intérprete. En las palabras del propio MACCORMICK, «la presencia de elementos no escritos parece ser una característica general del derecho» (MACCORMICK, 2005: 244).

\subsection{La lectura interpretativa de las fuentes del derecho}

En este momento, ya puede percibirse por qué la teoría interpretativa del derecho y de la decisión jurídica de MACCORMicK, que está en gran medida influenciada por la concepción de DwORKIN sobre el «derecho como integridad», está más allá de la 
formulación positivista de la denominada «Tesis de las Fuentes Sociales», que parece ser el único punto comúnmente aceptado por todos los positivistas.

Todos los positivistas concuerdan en que el criterio decisivo para determinar la validez de una norma jurídica es la fuente o el origen de esta norma. Cuando una regla puede ser reconducida a una fuente jurídicamente reconocida, los teóricos del derecho pueden identificarla con un grado razonable de seguridad. Incluso las formas no ortodoxas de positivismo, como las teorías «inclusivas» de WALUCHOW, del último HART y de COLEMAN, tienen necesariamente que aceptar que cualquier contenido normativo que derive de una fuente jurídica válida tiene el carácter de una norma jurídica, bastando que esta regla satisfaga a la regla-maestra establecida en un sistema jurídico particular como un test para la validez jurídica de sus normas.

El reconocimiento, por MACCORMICK, del carácter inmanentemente argumentativo del derecho, por otro lado, parece implicar una doctrina de las fuentes del derecho completamente diferente. Los materiales encontrados en las fuentes del derecho, como leyes, precedentes, tratados y actos administrativos, no son «auto-interpretativos y auto-aplicables» (MACCORMICK, 2005: 23). Bien entendidos, esos materiales son «derecho» únicamente en un sentido pre-interpretativo (MACCORMICK, 1998a). Las ideas de MACCORMicK sobre la teoría de los precedentes judiciales son un buen ejemplo para ilustrar este punto. MACCORMICK sostiene que las teorías del precedente basadas en el derecho natural o en el positivismo jurídico contribuyeron no sólo para diferentes interpretaciones de la doctrina del stare decisis en el Reino Unido, sino también para diferentes usos prácticos y aplicaciones del derecho jurisprudencial. De un lado, la así denominada «teoría declaratoria de la decisión judicial» — que se presenta como compatible con los puntos de vista del derecho natural y que dominó el escenario jurisprudencial de Gran Bretaña hasta el inicio del siglo XVIII- «fundamentaron una hostilidad en relación a cualquier doctrina de precedentes absolutamente vinculantes, basadas en el argumento de que es lógicamente posible la existencia de errores judiciales, y por tanto los precedentes son apenas declaratorios o constituyen una evidencia de lo que el derecho es, y no propiamente normas jurídicas» (MACCORMicK, 1998a: 182). Por otro lado, el positivismo, al enfatizar el aspecto social del derecho, «necesariamente niega las premisas de la teoría declaratoria»:

No hay una esencia del derecho más allá de lo que fue decidido como «derecho» por alguna autoridad competente. De esto se sigue que, si los precedentes constituyen una evidencia del derecho, sólo pueden serlo porque a los jueces explícita o implícitamente fue atribuida autoridad para crear derecho a través de sus decisiones. De modo inverso, el propio reconocimiento del precedente como una evidencia del contenido del derecho significa el reconocimiento del poder de los jueces para crear el derecho (MACCORMICK, 1998a: 183).

Podemos ver, por tanto, que estas teorías producen serias consecuencias normativas en el modo en que el precedente judicial es recibido y aplicado en la práctica jurídica. Es por causa de tales consecuencias que MACCORMICK, al tratar el tema del precedente, sostiene que es necesaria una teoría que pueda superar la dicotomía «derecho natural versus positivismo», pues esta es la única manera de trascender los límites de la teoría declaratoria del derecho y de su contraparte positivista:

Ya no es posible [...] contentarse con un simple contraste entre el derecho natural y el positivismo al tratarse del precedente. Esta diferencia fue planteada, principalmente, por 
R. DwORKIN, cuyo trabajo subvirtió la dicotomía sencilla entre el positivismo y el derecho natural. En lugar de un modelo de derecho como un sistema de reglas derivadas de fuentes predeterminadas, DwORKIN nos invita a re-concebir el derecho como un concepto esencialmente «interpretativo». Respecto de todo el conjunto de decisiones del legislador y de los jueces y de otras autoridades, a los cuales el positivismo conceptuó como agentes creadores del «sistema jurídico», DwORKIN nos invita a entenderlos como derecho únicamente en un sentido «pre-interpretativo» (MACCORMICK, 1998: 183).

Como lo explica MACCORMICK, de acuerdo con esta concepción la propia noción de «fuentes del derecho» precisa ser revisada: «Para un seguidor de la perspectiva de DwORKIN, si tratamos un precedente, o incluso un acto del Parlamento o del Ejecutivo, como una "fuente del derecho", la concepción de "fuente" en cuestión debe ser radicalmente diferente de la asumida por un modelo positivista» (MACCORMICK, 1998a: 183). Siguiendo los pasos de Dworkin, MACCormick llega a una teoría de las fuentes del derecho según la cual las leyes, los precedentes y demás materiales jurídicos «no constituyen en sí mismas derecho, sino únicamente materiales a partir de los cuales la tarea de construir el derecho debe realizarse». La especificación del contenido del derecho depende de un proceso interpretativo conducido por la idea de «integridad», en el sentido de DwORKIN (2000), o de «coherencia normativa», que es la misma idea en el vocabulario de MACCORMICK (2005). En dicha construcción interpretativa, el intérprete torna el derecho operativo cuando aplica esos materiales de acuerdo con los principios morales implícitos en el derecho, y este proceso hermenéutico es siempre guiado por la idea regulativa de alcanzar la mejor interpretación posible de esos materiales pre-interpretativos. Se puede ver, por tanto, que MACCORMICK defiende una teoría de las fuentes del derecho y una teoría de los precedentes que son incompatibles con el positivismo jurídico y con la visión de que la autoridad de una fuente es el único factor relevante para determinar la validez de una regla que pueda ser extraída de un precedente judicial. Las normas derivadas de los precedentes (o incluso de las leyes) serán fijadas en un discurso interpretativo donde los argumentos basados en la moralidad política y los argumentos basados en la razón práctica son ponderados de manera apropiada.

\subsection{La teoría de la argumentación jurídica de MACCORMICK}

El carácter argumentativo o discursivo del derecho y la concepción de fuentes del derecho como materiales pre-interpretativos, y no como respuestas definitivas a la cuestión de la validez de una regla, son profundamente compatibles con la ambición de MACCORMicK de construir una teoría normativa de la argumentación jurídica. Si el derecho necesariamente se refiere a una cierta concepción de justicia, y si su contenido último depende de una interpretación constructiva en el contexto de pretensiones de validez disputables para fundamentar normas individuales producidas en el proceso de aplicación del derecho, entonces es razonable sostener que una de las tareas de la teoría del derecho debe ser la de ofrecer un conjunto de directivas para ayudar a los juristas a «discriminar entre los mejores y los peores argumentos, es decir, entre los más o menos racionalmente convincentes» (MACCORMICK, 2005: vi). En este sentido, parte de la estrategia de MACCORMICK para reconciliar la dimensión argumentativa del derecho con el valor de la certeza del derecho - que son ambos supuestos e implicados por la idea política del Estado de derecho- es elaborar una teoría de la argumentación 
jurídica que pueda ser capaz de encontrar los mejores criterios de racionalidad para las decisiones judiciales y los requisitos fundamentales que se imponen sobre el proceso de argumentación jurídica.

Aunque mucho pueda decirse sobre la teoría de la argumentación jurídica de MACCORMICK, es suficiente para nuestros propósitos hacer referencia al universalismo kantiano que es aceptado por la versión final de su proyecto teórico. Para MACCORMICK, cualquier decisión jurídica sólo puede ser justificada si puede pasar un test de universalización. Eso se sigue de la propia idea de justificación: «justificar es demostrar que algo es correcto», y «demostrar que algo es correcto es demostrar que, desde cualquier punto de vista objetivo acerca del asunto, el acto debe ser realizado, o por lo menos debería haber sido realizado, en vista de las características y de las circunstancias del caso» (MACCORMICK, 2005: 98). No hay justificación sin universalización para la teoría de MACCORMicK sobre la argumentación jurídica:

Para que hechos particulares —o motivos particulares— constituyan razones justificativas deben ser subsumibles en un principio de acción universalmente establecido, aunque este universal pueda ser tenido como derrotable. Eso se aplica a la razón práctica de modo general, y al razonamiento jurídico como un departamento del razonamiento práctico (MACCORMICK, 2005: 99).

Ello es suficiente para mostrar que la teoría de la argumentación de MACCORMICK se dirige hacia un test de universalizabilidad en el sentido del imperativo categórico de KANT, aunque este test sea adaptado a la forma del ideal de PERELMAN sobre el «auditorio universal» (Perelman; TYTECA, 1969) o de la idea regulativa de HABERMAS de una «situación ideal de habla» (HABERMAS, 1997).

De todos modo, MACCORMICK inequívocamente reconoce la importancia del principio moral de universalizabilidad cuando expresamente admite que la última versión de su teoría de la argumentación jurídica se aparta del no-cognitivismo de Hume, que solía estar presente en sus escritos de juventud, en busca de una filosofía moral kantiana (MACCORMicK, 2005: 30). En este aspecto, la teoría de la argumentación jurídica de MACCORMicK tuvo que incorporar dos tesis sostenidas por ALEXY para fundamentar su pretensión de que los principios de la moralidad universalista kantiana son relevantes para la argumentación jurídica: la tesis de la «pretensión de corrección» y la «tesis del caso especial». Si el derecho es un caso especial del discurso práctico y, como tal, erige una pretensión de corrección, entonces las reglas básicas de la argumentación que se aplican sobre el discurso práctico son también válidas para el discurso jurídico. Los mismos principios discursivos que se pueden encontrar en la filosofía moral kantiana son también válidos para el discurso jurídico, ya que este es nada más que un caso especial del primero con una serie de constricciones institucionales ${ }^{3}$. Sin esas dos tesis, MACCORMICK no sería capaz de fundamentar su pretensión, también común a ALEXY, de que las reglas de argumentación propuestas por pensadores como KANT o HABERMAS son también aplicables en la argumentación jurídica. Por tanto, no es difícil notar

3 Eso no significa obviamente, por otra parte, que el derecho y la moral no puedan distinguirse. El hecho de que el discurso jurídico opere en el interior de un conjunto de balizas institucionales implica que las decisiones jurídicas están limitadas por la exigencia de respeto a las leyes, a los precedentes y a otros materiales autoritativos que se encuentran en un sistema jurídico. Es esta constricción del razonamiento jurídico práctico lo que hace de él un caso especial del razonamiento práctico general. 
que la teoría de MACCORMICK sobre la argumentación jurídica — por lo menos en su forma más desarrollada - presupone una ruptura con la tradición positivista. Como intentaré demostrar en las secciones finales de este trabajo, las tesis del «caso especial» y de la «pretensión de corrección», en la forma como MACCORMICK las interpreta, no pueden ser conciliadas con ningún tipo de positivismo.

\subsection{La tesis de la pretensión de corrección}

Uno de los momentos en que MACCORMICK más se aparta del positivismo es aquel en el que sostiene que el derecho erige una pretensión implícita de justicia. En efecto, MACCORMICK argumenta que el discurso jurídico se caracteriza por la presencia de ciertas pretensiones implícitas que están «necesariamente conectadas a la realización de interacciones discursivas entre la creación del derecho, el judiciario y las instituciones o agencias ejecutivas del Estado» (MACCORMICK, 2007: 274). Todo acto de habla que introduce una norma o ejercita algún tipo de autoridad jurídica está conectado con el acto ilocucionario de afirmar la corrección de las normas generales o individuales producidas por medio de este acto. Cualquier acto de habla realizado en el contexto de una argumentación jurídica envuelve ciertas condiciones de fondo o pretensiones implícitas, y en particular la pretensión de corrección jurídica y moral (MACCORMICK, 2007: 275). Entendido como orden institucional normativo, el derecho busca la justicia y necesariamente se dirige a solucionar conflictos prácticos de modo moralmente aceptable: «Una cierta pretensión de justicia, es decir, una aspiración afirmada de estar alcanzando la justicia (aún si dicha pretensión fuera la máscara para una intención partidaria o siniestra) está necesariamente incorporada en el propio acto de creación del derecho en el contexto de un Estado-nación» (MACCORMICK, 2007: 276).

La pretensión de corrección incluye una pretensión de corrección moral, aunque no se contente exclusivamente con ella, y no sólo una pretensión de corrección de acuerdo con las leyes positivas del Estado. Dicha pretensión, que juega también un papel central en la teoría de ALEXY sobre la argumentación jurídica, está íntimamente conectada con la idea de que el derecho posee un carácter argumentativo. Los fundamentos de esta tesis pueden ser encontrados en la filosofía del lenguaje de HABERMAS. En su conocido ensayo Teorías de la Verdad, HABERMAS ha criticado las teorías filosóficas que definen la verdad de una aserción como la «correspondencia» con una cierta cosa o estado de cosas que es probado como existente en un mundo de objetos físicos (HABERMAS, 1997). Contrariamente a estas teorías, HABERMAS sostiene una concepción de verdad como consenso racional, que puede ser sintetizada en el párrafo siguiente:

La verdad no es una propiedad de las informaciones, sino de los enunciados; se mide no por la probabilidad de cumplimiento de pronósticos, sino por la unívoca alternativa de si la pretensión de validez de las afirmaciones es discursivamente desempeñable o no lo es. Llamamos verdaderos a los enunciados que podemos fundamentar...

Sólo puedo (con la ayuda de oraciones predicativas) atribuir un predicado a un objeto si también cualquiera que pudiera entrar en discusión conmigo atribuyese el mismo predicato al mismo objeto; para distinguir los enunciados verdaderos de los falsos, me refiero al juicio de los otros y, por cierto, al juicio de todos aquellos con los que pudiera iniciar una discusión (incluyendo contrafácticamente a todos los oponentes que pudiera encontrar si mi vida fuera coextensiva con la historia del mundo humano) (HABERMAS, 1997: 171). 
Con este discurso ideal, HABERMAS busca el criterio de verdad que acredita estar ausente en las teorías epistemológicas que identifican la verdad de una aserción con la mera correspondencia, mediada por la experiencia sensorial, con un cierto estado de cosas. Ya que todos tenemos diferentes experiencias, una concepción de verdad basada únicamente en la percepción sensorial estaría equivocada porque ella no puede garantizar la objetividad del conocimiento que ella produce. Si no hay una realidad objetivamente accesible a nuestros sentidos, la racionalidad de las expresiones utilizadas por A o por B sólo puede ser verificada de acuerdo con la justificabilidad discursiva de las pretensiones de validez contenidas en los actos de habla desempeñados por cada hablante. Dicho en otras palabras, al comprender el discurso como un procedimiento argumentativo, HABERMAS está sosteniendo que en las interacciones lingüísticas entre A y $\mathrm{B}$, «ambos erigen pretensiones con sus expresiones simbólicas, las cuales precisan ser criticadas y defendidas, es decir, fundamentadas» (HABERMAS, 1984: 9). Un juicio - sobre la verdad, en el caso de los actos de habla constatativos, o sobre la corrección, en los actos de habla regulativos, donde lo que se discute no es la verdad de un hecho, sino la validez de una norma- (HABERMAS, 1997: 130) sólo puede ser objetivo «si se realiza sobre la base de una pretensión de validez trans-subjetiva», de modo que las «aserciones y acciones dirigidas para fines son tanto más racionales cuanto más la pretensión que está conectada a ellas pueda ser defendida contra la crítica» (HABERMAS, 1984: 6). Por tanto, HABERMAS necesita adoptar un concepto de racionalidad comunicativa que está basado en un discurso cuyas propiedades formales sean capaces de generar un consenso libre y unificador.

Igual que los enunciados sobre hechos, las acciones normativamente reguladas también contienen expresiones significativas que están conectadas con pretensiones de validez criticables. Las normas a que estas acciones se refieren pueden también ser intersubjetivamente reconocibles, y la conveniencia discursiva de las pretensiones de validez erigidas para estas normas es lo que determina su racionalidad (HABERMAS, 1984: 15-16). En el campo de la ética, HABERMAS adopta una posición cognoscitivista según la cual las cuestiones prácticas o normativas pueden ser solucionadas por medio de una argumentación aducida en un discurso práctico donde la corrección de una norma es examinada (HABERMAS, 1984: 19). Este tipo de discurso es un proceso comunicativo que sólo puede ser racional si se aproxima a las siguientes condiciones ideales: 1) los hablantes deben estar en una «situación ideal de habla» que es fundamentalmente caracterizada por la «simetría de condiciones», de modo que cada participante en el discurso puede estructurar sus actos de habla de modo que no haya más coerción que la proveniente de la fuerza racional de los mejores argumentos (HABERMAS, 1984: 25); 2) la argumentación, como proceso de comunicación, debe ser entendida como «una forma de interacción sujeta a reglas especiales», y 3) la argumentación «tiene como finalidad producir argumentos vinculantes que sean convincentes en virtud de sus propiedades intrínsecas y respecto de los cuales ciertas pretensiones de validez pueden ser redimidas o rechazadas» (HABERMAS, 1984: 35).

Ya que no puedo profundizar aquí en la teoría del discurso racional, me voy a limitar a enfatizar uno de los puntos que aparentan ser centrales para ALEXY y MACCormicK: la idea de que los actos de habla regulativos siempre erigen una pretensión de corrección normativa. Es con base en esta premisa que se sostiene que en todo acto 
de creación y aplicación del derecho se erige implícitamente una pretensión de que dicho acto es correcto. En el núcleo de esta pretensión se encuentran: 1) la aserción de que el acto jurídico es substancial y procesalmente correcto; 2 ) la pretensión (que genera una garantía) de justificabilidad de esta aserción, y 3 ) la expectativa de aceptabilidad de la corrección por todos los destinatarios de la norma jurídica (ALEXY, 1998: 208). ¿Pero cómo puede AleXY justificar esta tesis? Responde a esta cuestión de la siguiente manera: «Una pretensión implícita puede ser explicitada si se demuestra que su negación expresa es absurda» (ALEXY, 2005: 21). Adopta, por tanto, una estrategia pragmático-formal de demostrar que la negación explícita de la pretensión de corrección implica una contradicción entre el contenido del acto jurídico (sea una ley, una decisión judicial, etc.) y el contenido de la pretensión implícita en su promulgación. ALEXY denomina a este tipo de contradicción «contradicción performativa» (ALEXY, 1998: 210). Todo participante en el discurso jurídico que expresamente niegue la pretensión de corrección comete una contradicción de este tipo. Sería una contradicción performativa, por ejemplo, si en la promulgación de una constitución el primer artículo expresamente afirmase que «X es un Estado injusto». Lo mismo ocurriría si un juez pronunciara una sentencia enunciando algo como «X es condenado a prisión, aunque eso sea una interpretación incorrecta del derecho». La decisión de este juez podría ser clasificada como absurda porque contendría tanto una aserción implícita de que la decisión es correcta como una aserción explícita que contradice la parte implícita.

ALEXY sostiene, en uno de los fragmentos más importantes de su argumentación, que la pretensión de corrección tiene la función de establecer una conexión necesaria entre el derecho y la moral. La pretensión de corrección atribuye al derecho un carácter ideal que no estaba presente en las descripciones positivistas según las cuales el derecho puede ser definido como mera facticidad o como el resultado del ejercicio de la autoridad. Esta dimensión ideal del derecho fundamenta un principio general de moralidad que tiene la fuerza de una norma jurídica implícita en todo sistema jurídico. Juntamente con esta pretensión, ALEXY reconoce una norma pragmáticamente supuesta que establece un deber de interpretar y aplicar las normas jurídicas de forma

correcta. Como consecuencia de la pretensión de corrección, puede argüirse que hay una conexión argumentativo-metodológica entre el derecho y la moral (ALEXY, 1998) que implica que los aplicadores del derecho tienen la obligación prima facie de hacer «justicia de acuerdo con el derecho». Esto es precisamente lo que MACCORMICK quiere decir cuando sostiene que el derecho está «necesariamente enfocado hacia la justicia», y esta pretensión está obviamente en contradicción con el positivismo jurídico. En resumen, dicha pretensión atribuye al derecho un carácter aspiracional e introduce una distinción entre el «derecho positivo real» (actual positive law) y el «derecho positivo ideal» (ideal positive law) (MACCORMICK, 2007: 257), así como una obligación discursiva, para los aplicadores del derecho, de intentar reconciliar esas dos dimensiones en sus interpretaciones constructivas.

\subsection{La tesis del caso especial}

Es necesario pronunciarse también sobre la tesis de que la argumentación jurídica es un caso especial de discurso práctico. La tesis del caso especial es relevante para las 
teorías de la argumentación jurídica porque esta hace más fácil la percepción de las presuposiciones pragmático-formales del discurso jurídico. Cuando decimos que el discurso jurídico es un caso especial del discurso práctico, nos comprometemos con la aserción de que hay ciertas obligaciones discursivas para los participantes de este tipo de discurso. Cualesquiera que sean las constricciones aplicables al discurso práctico general, también serán válidas para el discurso jurídico, aunque con el requisito adicional de que los hablantes deben obedecer a las leyes positivas con las cuales ellos operan. Como MACCORMICK escribe al expresar su concordancia con la tesis del caso especial, en la formulación de ALEXY, «la argumentación jurídica debe ser entendida como un caso especial de razonamiento práctico, y debe por tanto conformarse a las condiciones de racionalidad y de razonabilidad que se aplican a todos los tipos de razonamiento práctico» (MACCORMICK, 2005: 17).

La tesis del caso especial es, por consiguiente, importante porque conecta el derecho, la racionalidad y la retórica. También implica ciertos «deberes comunicativos» (VIEHWEG, 1979) para cualquiera que entre en un discurso o erija una pretensión jurídica. Por consiguiente, la adhesión de MACCORMICK a la tesis del caso especial implica que las razones morales, políticas y pragmáticas que inevitablemente se aplican en el discurso práctico general sean necesariamente incluidas en la argumentación jurídica, lo que contradice todo lo que los positivistas afirman sobre el razonamiento jurídico ${ }^{4}$.

\subsection{La eticidad de las instituciones jurídicas}

Finalmente, la teoría institucionalista de MACCORMICK es también substancialmente diferente del positivismo porque se niega a aceptar la descripción del derecho como un sistema necesariamente formal y monolítico de reglas previamente dadas. En este sentido, me parece que la mayor parte de los positivistas contemporáneos están tan comprometidos con sus prejuicios metodológicos que normalmente no consiguen siquiera percibir uno de los puntos más importantes de la teoría institucionalista de MACCORMICK, que se relaciona con la eticidad de las instituciones jurídicas (BANKOWSKI, 2007). Con base en una lectura «selectiva» de los textos de MACCORMICK, resaltan algunos «sentidos comunes» en la teoría institucional del derecho y terminan sosteniendo que encuentran en MACCORMICK un aliado en lugar de un oponente ${ }^{5}$. Cuando hacen esto, sin embargo, tienden a tergiversar la teoría que están analizando. En efecto, la teoría institucional de MACCORMICK presupone un elemento de entendimiento mutuo entre los miembros de una comunidad jurídica que está intrínsecamente sedimentado en el derecho (MACCORMICK, 1998b: 305). El derecho emana de la interacción social de los miembros de dicha comunidad, que es mediada por un proceso discursivo

\footnotetext{
${ }^{4} \mathrm{Si}$ aceptamos este argumento, tenemos que afrontar nuevos problemas como, por ejemplo, el del potencial conflicto entre argumentos «positivos» en sentido estrecho y los principios de justicia que pueden fundamentarse por medio de las reglas del discurso práctico. Ni MACCORMICK ni ALEXY parecen aceptar que haya siempre una primacía incondicionada de las razones «positivas» sobre las demás. Todavía no estamos en condiciones, sin embargo, de afrontar ese problema en el presente trabajo.

5 Se pueden encontrar ejemplos de positivistas que intentan leer a MACCORMICK como perteneciente a su campo de discusión en SCHAUER (1996), WALUCHOW (1994) y Villa (2009).
} 
en que estos miembros ejercen influencia unos sobre los otros y son transformados por las experiencias que adquieren en dichas interacciones. Este «entendimiento mutuo» no puede ser de cualquier tipo, ya que lo que hace a una regla institucionalmente reconocible no es sólo la conformidad con ciertas «reglas constitutivas» que determinan su significado - como acontece, por ejemplo, en el modelo de reglas constitutivas de SEARLE (1970)—, sino los «principios subyacentes» que constituyen la «causa final» de una institución concreta (MACCORMICK, 1998b: 305). Al igual que DwOrKIn, MACCORMICK acredita que el significado de la práctica social denominada «derecho» deriva en parte de principios que proveen la coherencia general del sistema jurídico y que pertenecen al tiempo a la moralidad y al derecho positivo.

La distinción entre la posición de MACCORMICK y la del positivismo angloamericano es clara cuando se compara la pretensión positivista de tratar el derecho como «un sistema contenido en sí mismo y autogenerador que necesita ser distinguido de la política para organizar nuestras vidas» (BANKOWSKI, 2007: 198) con la idea constructivista, que MACCORMICK explícitamente acepta, según la cual el derecho está abierto a discursos de aplicación que llevan a su adaptación y eventual reinterpretación con base en consideraciones éticas y morales (GÜNTHER, 1993; MACCORMICK, 2005: 237 y ss.). Según la mejor interpretación de la teoría institucional de MACCORMICK, el derecho y sus instituciones crean una especie de espacio ético donde la coordinación para el bien común se hace posible y donde los individuos que viven en una comunidad política pueden participar en la formación de los valores y de los principios que rigen sus vidas (BANKOWSKI, 2007: 202). Las instituciones jurídicas son consideradas valiosas y los miembros de la comunidad política reconocen sus leyes no meramente como un conjunto cierto y previsible de órdenes expedidos sin su participación, sino como un sistema normativo que expresa una forma de vida a la cual ellos pertenecen y que es constitutiva para sus identidades y sus auto-comprensiones. Por otro lado, como BANKOWSKI argumenta de forma convincente, el positivismo tiende a alienar a los individuos en una sociedad capitalista y a reducirlos a la condición de jugadores en un juego de reglas donde la creatividad y el sentido crítico son restringidos (narrowed down) (BANKOWSKI, 2007: 199).

Ninguno de esos efectos negativos, sin embargo, está presente en la teoría de MACCormicK. Las Instituciones del derecho de MACCORMICK son en realidad un intento de reconciliar de forma crítica la teoría jurídica y la teoría institucional, la teoría y la práctica, el derecho y su justificación moral, la política y la eticidad de la comunidad en la cual el derecho es constantemente reconstruido. El núcleo de este proyecto teórico, en mi interpretación, consiste en trascender a los límites del pensamiento jurídico que los positivistas establecen para sí mismos.

\section{CONCLUSIÓN}

Se puede percibir en esta discusión que el post-positivismo es una alternativa genuina al positivismo, pues rechaza los puntos centrales de este tipo de teoría y favorece una lectura interpretativa de las fuentes del derecho que evita el esencialismo empírico que de modo general afecta al positivismo. 
Desde el momento en que se reconoce que el derecho es un concepto argumentativo y que el discurso práctico general está inherentemente conectado a la argumentación jurídica, debido a que los argumentos morales pueden volverse obligatorios en el discurso jurídico —al menos en los denominados «casos difíciles»-, ya no puede aceptarse la distinción nítida entre «pura creación» y «pura aplicación» del derecho. Tampoco puede aceptarse la distinción entre «teorías del derecho» y «teorías de la decisión», pues en muchos casos la admisión de argumentos morales en el razonamiento jurídico deriva de la propia naturaleza del derecho. Esas conclusiones pueden muy bien ser un camino que nos va a llevar lejos del positivismo jurídico.

\section{REFERENCIAS BIBLIOGRÁFICAS}

AleXY, R., 1998: «Law and Correctness», en Current Legal Problems, vol. 51, 205-221.

- 2005: «Derecho y moral», en La institucionalización de la justicia, Granada: Comares, 17-29.

AtienzA, M., 2006: «Entrevista a Neil MacCormick», Doxa - Cuadernos de Filosofía del Derecho, vol. 29, 479-489.

BANKOwSKI, Z., 2007: «Bringing the Outside in the Ethical Life of Legal Institutions», en T. GizBerT-STUDNiCKI y J. Stelmach, Law and legal cultures in the $21^{\text {st }}$ century, Varsóvia: Oficyna, 193-216.

BoвBIO, N., 1998: «Sur le positivisme juridique», en Essais de theorie du droit, Paris: LGDJ, 23-38.

Coleman, J., 2007: «Beyond the Separability Thesis: Moral Semantis and the Methodology of Jurisprudence», Oxford Journal of Legal Studies, vol. 27, 581-608.

DwORKIN, R., Law's Empire, 11. imp., Cambridge, MA: Belknap.

GÜNTHER, K., 1993: The sense of appropriateness. Application discourses in morality and in Law, trad. de John Farrel, Albany: State University of New York Press.

Habermas, J., 1984: The Theory of Communicative Action, Vol. 1. Reason and the Rationalization of Society, trad. Th. McCarthy, Boston: Beacon Press.

- 1997: «Teorías de la Verdad», en Teoría de la acción comunicativa: complementos y estudios previos, trad. de M. Jiménez Redondo, Madrid: Cátedra, 113-158.

MACCORMicK, N., 1978: Legal Reasoning and Legal Theory, Oxford: Clarendon.

- 1998a: «Precedent as a Source of Law», en E. ATTWOOL y ComANDuCCI, Sources of Law and Legislation: Proceedings of the 17th World Congress of Philosophy of Law and Social Philosophy (IVR), Bologna, June 16-21, 1995, Archiv für Rechts und-Sozialphilosophie, Beiheft 69, 177-185.

- 1998b: «Norms, institutions and institutional facts», Law and Philosophy, vol. 17, 301-345.

- 2005: Rhetoric and the Rule of Law. A Theory of Legal Reasoning, Oxford: Oxford University Press.

- 2007: Institutions of Law, Oxford: Oxford University Press.

Perelman, Ch., y OlBrechts-TyteCA, L., 1970: Traité de l'argumentation: La nouvelle rhétorique, Bruxelas: Université Libre de Bruxelles.

RAZ, J., 1994: Ethics in the public domain, Oxford: Oxford Univeristy Press.

Schauer, F., 1996: «Positivism as Pariah», en R. GeOrge, The Autonomy of Law: Essays on Legal Positivism, Oxford: Oxford University Press, 32-56. 
SEARLE, J. R., 1970: Speech Acts. An essay in the Philosophy of Language, Cambridge: Cambridge University Press.

VIEHWEG, Th., 1979: Tópica e jurisprudencia, trad. de T. Sampaio Ferraz Júnior, Brasília: Departamento de Imprensa Nacional.

VilLA, V., 2009: «Neil Maccormick’s Legal Positivism», en M. Del Mar y Z. BANKowski, Law as Institutional Normative Order, Aldershot: Ashgate, 44-64.

WaLuCHOW, W., 1994: Inclusive Legal Positivism, Oxford: Clarendon. 